\title{
EVALUANDO LA INFLUENCIA DE LA DISPERSIÓN URBANA EN LA ELECCIÓN DE MODOS DE TRANSPORTE
}

\author{
Olta Braçe \\ Grupo de Investigación Health \& Territory Research (HTR), Universidad de Sevilla \\ oltabrace@msn.com \\ Marco Garrido Cumbrera \\ Departamento de Geografía Física y Análisis Geográfico Regional, Universidad de Sevilla \\ mcumbrera@us.es \\ David Gálvez Ruiz \\ Departamento de Estadística e Investigación Operativa Universidad de Sevilla \\ davidgalvez@us.es \\ Enrique López Lara \\ Departamento de Geografía Física y Análisis Geográfico Regional, Universidad de Sevilla \\ elopezl@us.es
}

\section{RESUMEN}

La promoción del transporte público y de modos de transporte no motorizados tiene como objetivo reducir la dependencia del vehículo privado. Muchos estudios, realizados principalmente en países anglosajones, han analizado la posible relación entre la dispersión urbana y el uso de modos de transporte, aunque a escala metropolitana. Sin embargo, la evidencia de esta relación no ha sido suficientemente evaluada en países mediterráneos. El objetivo del presente estudio es evaluar, de manera empírica, la influencia de la dispersión urbana en la elección de modos de transporte específicos a escala sub-municipal.

Palabras clave: dispersión urbana, modos de transporte, transporte no motorizado, regresión ordinal PLUM.

Fecha de recepción: mayo 2016.

Fecha de aceptación: junio 2017.

1 Los resultados de este artículo forman parte del Proyecto de I+D "Evaluación del Impacto de la Dispersión Urbana en los Hábitos de Vida, la Movilidad Urbana y la Salud de la Población Adulta en Áreas Metropolitanas Españolas (URDIS)", financiado por el Ministerio de Economía y Competitividad de España (CSO2014-59524-P). 


\section{ABSTRACT}

The promotion of public transport and active commuting modes is aimed to reduce private vehicle dependence. Many studies, conducted mainly in Anglo-Saxon regions have investigated the relationship between sprawl and the use of transport modes, albeit using the metropolitan scale as a spatial ambit of study. However, evidence of this relationship has been poorly assessed in Mediterranean countries. The objective of this study is to assess, in an empirical way, the influence of urban sprawl on the choice of specific commuter transport modes at sub-municipal scale.

Keywords: urban sprawl, modes of transport, active commuting, ordinal regression PLUM.

\section{INTRODUCCIÓN}

El rápido y constante crecimiento de la población y del espacio urbano son fenómenos que acontecen en todo el mundo (Glaeser, 2011), estimándose que en el 2020, el 80\% de la población europea residirá en zonas urbanas (Eurostat, 2012). Gran parte de este crecimiento urbano se caracteriza por un patrón denominado "mancha urbana" (Duany, Plater-Zyberk, and Speck, 2001), proceso económico y social asociado con la baja densidad residencial y la dependencia del vehículo privado (Ewing, 2008).

Los fuertes incrementos de la producción y de los intercambios, junto al aumento de la dimensión de las ciudades, se han ido produciendo paralelamente a la transformación de la economía mundial de agrícola y rural a urbana e industrial, generando un impacto ambiental cada vez de mayor intensidad y extensión territorial (Burgess, 2003).

Debido a los procesos de expansión urbana acaecidos en las últimas décadas en la Europa mediterránea, la morfología urbana de sus ciudades ha experimentado importantes transformaciones, convirtiendo el fenómeno de la dispersión urbana en un tema central, especialmente en lo referente a la planificación territorial sostenible. La dispersión urbana está reconocida como un problema serio de diseño urbano que conduce a la pérdida de espacios naturales. Además, supone un alto coste en infraestructuras y energía, incita a la segregación de usos del suelo, aumenta la necesidad de desplazamientos y la dependencia del vehículo privado. Todo ello está provocando el aumento de la congestión del tráfico, del consumo energético y de la contaminación (Commission of the European Communities, 2004).

El fenómeno de la dispersión urbana tuvo su boom con la aparición del coche y su uso masivo por parte de todas las capas sociales. Al mismo tiempo, se producía una disminución de la densidad residencial a lo largo de la periferia de las grandes ciudades, reflejando cambios en las preferencias del mercado: gran parte de la población se trasladó a la periferia atraída por viviendas de mayor tamaño, en áreas de menor densidad, no estando dispuesta a pagar más por vivir cerca del lugar de trabajo. A su vez, las constructoras e inmobiliarias han aprovechado esa demanda para generar nuevas áreas urbanas (Frumkin, Frank, y Jackson, 2004).

Históricamente, el crecimiento de las ciudades se ha debido principalmente al aumento de la población urbana. Sin embargo, en las últimas décadas, a pesar de la escasa presión 
demográfica, diversos factores han impulsado el crecimiento urbanístico. Las causas que explican la tendencia de expansión de las ciudades son variadas y complejas, e incluyen el aumento de la calidad y cantidad de infraestructuras y de los sistemas de transporte, el acceso al vehículo privado, el menor precio del suelo, la menor contaminación acústica y atmosférica, nuevas preferencias individuales a residir en viviendas unifamiliares, o cambios en la estructura familiar con mayor presencia de familias unipersonales (European Environment Agency, 2006). A ello se suma una baja densidad (mayor consumo de suelo por hogar o lugar de trabajo), una mono-especialización de los usos del suelo, escasa jerarquización del espacio, un espacio entorpecido por barreras, la falta de atractivo estético y, en definitiva, una pauperización del paisaje (Marmolejo Duarte y Stallbohm, 2008).

El fenómeno de la dispersión urbana es más complejo que el simple crecimiento de las ciudades, pues se basa en el éxodo de la población desde el centro hacia la periferia, aumentando la población que reside en áreas periféricas frente a aquella que lo hace en el núcleo central (Bruegmann 2005).

Durante la primera década de este siglo, y hasta la crisis del año 2008, España ha experimentado uno de los mayores auges de la construcción de los países de alta renta. Este boom de la vivienda supuso uno de los principales motores del crecimiento económico de España (Akin et al., 2014), permitiendo un aumento espectacular de los niveles de motorización y la expansión de las aglomeraciones urbanas. En 2006, la Agencia Europea de Medio Ambiente alertaba acerca del ritmo vertiginoso de crecimiento de las áreas urbanas, disociado del crecimiento demográfico y la proliferación de áreas urbanas dispersas, especialmente en países mediterráneos como España, Portugal y algunas partes de Italia (European Environment Agency, 2006).

El análisis transporte-territorio es complejo dada la intensidad y la variedad de las relaciones desarrolladas entre ambos conceptos a lo largo del tiempo, adquiriendo en cada etapa variables y consecuencias distintas (López Lara et al., 2003). El impacto más controvertido del fenómeno de la dispersión urbana es su influencia en la elección de modos de transporte específicos (Travisi, Camagni, and Nijkamp 2010). La proliferación de áreas urbanas de baja densidad, con predominio de usos de suelo segregados y escasez de servicios en proximidad, ha aumentado las distancias al área urbana central (Zhao et al. 2010), reduciendo las posibilidades para realizar desplazamientos no motorizados (Bahrainy y Khosravi, 2013; Brown et al., 2013; Ewing et al., 2014; García-Palomares, 2010; Jerrett et al., 2013).

La morfología urbana puede determinar la localización de servicios, de infraestructuras de transporte, y la movilidad de las personas. Los patrones de desarrollo urbano están ligados a la localización de los servicios y a la movilidad urbana. Del mismo modo, la movilidad urbana no puede considerarse independientemente de la morfología urbana (Newman y Kenworthy, 1999; UN-Habitat, 2012). Así, el modelo territorial urbano compacto facilita la implantación del transporte público y promueve la movilidad no motorizada, aportando beneficios desde el punto de vista económico, social y medioambiental. Por el contrario, el crecimiento urbano disperso fomenta la utilización y la dependencia del vehículo privado en los desplazamientos diarios (Rode y Floater, 2014; Giles-Corti et al., 2013).

El aumento del fenómeno de la dispersión urbana ha tenido repercusiones también en la distribución territorial y accesibilidad a los servicios y equipamientos. En efecto, en las áreas 
más dispersas existe menor presencia de servicios y equipamientos, lo que fomenta el uso del vehículo privado en la vida diaria (Braçe, 2016). En áreas urbanas dispersas se reducen las densidades y aumentan las distancias, resultando complejo y costoso establecer modos de transporte colectivo que resulten económicamente viables y atractivos para la población.

Influenciada por los principios de Desarrollo Sostenible, la política de transporte urbano se ha ido transformando, desde la adaptación de la ciudad al vehículo privado hacia la reducción de su uso, buscando nuevas actuaciones urbanísticas que limiten el crecimiento urbano descontrolado y el tráfico del automóvil y, al mismo tiempo, promuevan los modos de transporte sostenibles.

Gran parte de los análisis de la movilidad cotidiana se han centrado en el ámbito urbano, ya que el desplazamiento cotidiano por motivos laborales es considerado un indicador de integración de las áreas metropolitanas. En la medida en que las áreas metropolitanas se expanden, se diversifica también la movilidad diaria por la creación de nuevos lugares de residencia y las diferentes formas de desplazamientos pendulares que provocan.

El objetivo de esta investigación es evaluar de forma empírica la influencia que ejerce la dispersión urbana en la elección de modos de transporte específicos para los desplazamientos cotidianos, desde el lugar de residencia hasta el lugar de trabajo.

\section{METOdOLOGÍA}

\section{II.1. Diseño de estudio}

El ámbito territorial de esta investigación se corresponde con el municipio de Mairena del Aljarafe, situado en el Área Metropolitana de Sevilla, y cuenta actualmente con un total de 44.388 habitantes (2015). Este municipio de referencia fue dividido en 13 áreas homogéneas, utilizando para ello criterios urbanísticos y territoriales, eliminando del estudio tres áreas debido a su escasa población.

La información sobre el uso de modos de transporte fue extraída de la encuesta de "Movilidad urbana, hábitos de vida y salud de Mairena del Aljarafe 2015”. Para ello, se seleccionó una muestra de 505 personas (16-64 años) representativa por edad, sexo y área geográfica, a las cuales se realizó una entrevista personal en su propio domicilio. En dicha encuesta participaron cuatro encuestadores que recibieron formación específica para aumentar el grado de consistencia entre los resultados.

\section{II.2. Análisis estadísticos}

Para evaluar los niveles de dispersión de cada una de las áreas, se creó un índice compuesto de dispersión urbana constituido por 6 indicadores simples: densidad de población, densidad neta, coeficiente de edificación, tipo de usos de suelo, uso residencial predominante, y coeficiente de antigüedad constructiva. A cada indicador se le asignó un peso mediante el Proceso Analítico Jerárquico (AHP) y se calculó el índice compuesto de dispersión utilizando como agregador la Integral de Choquet (Gálvez Ruiz et al., 2017). En la Tabla 1 se presenta el índice de dispersión urbana resultante para cada área. 
Tabla 1

ÍNDICE DE DISPERSIÓN URBANA DE CADAÁREA UTILIZANDO LA INTEGRAL DE CHOQUET

\begin{tabular}{|c|c|c|}
\hline Área & Índice de dispersión urbana & Grado de dispersión \\
\hline Almendral y Java & 0,727 & Alto \\
\hline Centro Sur & 0,490 & Medio \\
\hline Simón Verde & 0,482 & Medio \\
\hline Centro Norte & 0,442 & Medio \\
\hline Estacada de Marqués & 0,440 & Medio \\
\hline Ciudad Expo & 0,400 & Bajo \\
\hline Cavaleri & 0,341 & Bajo \\
\hline Ciudad Aljarafe & 0,338 & Bajo \\
\hline Lepanto & 0,303 & Bajo \\
\hline Centro Histórico & 0,289 & \\
\hline
\end{tabular}

Fuente: elaboración propia.

Para comprobar la posible relación entre la dispersión urbana y el uso de determinados modos de transporte se utilizó el modelo de regresión PLUM, apto para variables categóricas ordinales. El análisis de Regresión Logística Multinomial utilizado en la mayoría de estudios de esta índole (Ewing \& Cervero, 2001; Ewing, Handyb et al, 2006, Ewing, Schmid et al, 2008; Ewing, Meakins et al, 2014) introduce las variables una a una, mientras se comprueba el efecto de cada variable independiente introducida en la variable explicada. Sin embargo, tanto la variable "grado de dispersión urbana" como especialmente "modo de transporte" (nomotorizados, transporte público y motorizados) para desplazarse al trabajo, se deben considerar como variables categóricas ordinales, teniendo en cuenta su relación con la actividad física y la sostenibilidad. Para ello, se ha utilizado como variable dependiente (o explicada) "modos de transporte" y como variable independiente o (explicativa) "dispersión urbana". De esta forma, para conservar la información contenida en la ordenación, cuando se ha analizado la influencia de la dispersión urbana sobre la probabilidad de utilizar un modo de transporte concreto, se aplicó la Regresión Ordinal (PLUM) mediante el paquete estadístico SPSS.

\section{RESULTADOS}

Las características sociodemográficas de los individuos que forman la muestra aparecen recogidos en la tabla 2. La población entrevistada de edad comprendida entre los 16 y los 64 años, se encontraba en su mayoría trabajando, presentando un alto porcentaje de estudios universitarios, residían en viviendas unifamiliares mayoritariamente y, en una alta proporción, contaba con vehículo privado (coche o moto).

Para evaluar las pautas en los desplazamientos diarios de la población encuestada en cada una de las áreas delimitadas, se agruparon los modos de transporte distinguiendo entre desplazamientos motorizados (coche y/o moto), transporte público (autobús y/o metro) y nomotorizados (caminar o bicicleta).

En el Mapa 1 se puede distinguir que cuanto mayor es el porcentaje de la población que utiliza modos de transporte motorizados en sus desplazamientos diarios, mayor es el grado 
de dispersión de las áreas (como Almendral y Java, Centro Sur y Simón Verde), mientras que, por su parte, donde menos se utilizan los modos de transporte motorizados es en las áreas con menor grado de dispersión urbana (es decir, en Lepanto, Ciudad Aljarafe y Cavaleri).

Tabla 2

CARACTERISTICAS SOCIODEMOGRÁFICAS DE LA MUESTRA ENCUESTADA EN EL MUNICIPIO DE MAIRENA DEL ALJARAFE

\begin{tabular}{|l|c|}
\hline \multicolumn{1}{|c|}{ Variables socio-demográficas } & Media \pm DE I \% (n) \\
\hline Rango de edad & $16-64$ \\
\hline Edad media & $40 \pm 13$ \\
\hline Género: Mujer & $50 \%(254)$ \\
\hline Nivel de Estudios: Universitarios & $38 \%(192)$ \\
\hline Estado Civil: Casado & $61 \%(308)$ \\
\hline Situación Laboral: Empleado & $58 \%(295)$ \\
\hline Tipología de Viviendas: Unifamiliares & $52 \%(262)$ \\
\hline Vehículos: Coche o Moto & $77 \%(388)$ \\
\hline & $\mathbf{N}: \mathbf{5 0 5}$ \\
\hline
\end{tabular}

Mapa 1

DISTRIBUCIÓN DE LOS MODOS DE TRANSPORTE UTILIZADOS EN LOS DESPLAZAMIENTOS DIARIOS EN LAS ÁREAS DEL MUNICIPIO DE MAIRENA DEL ALJARAFE

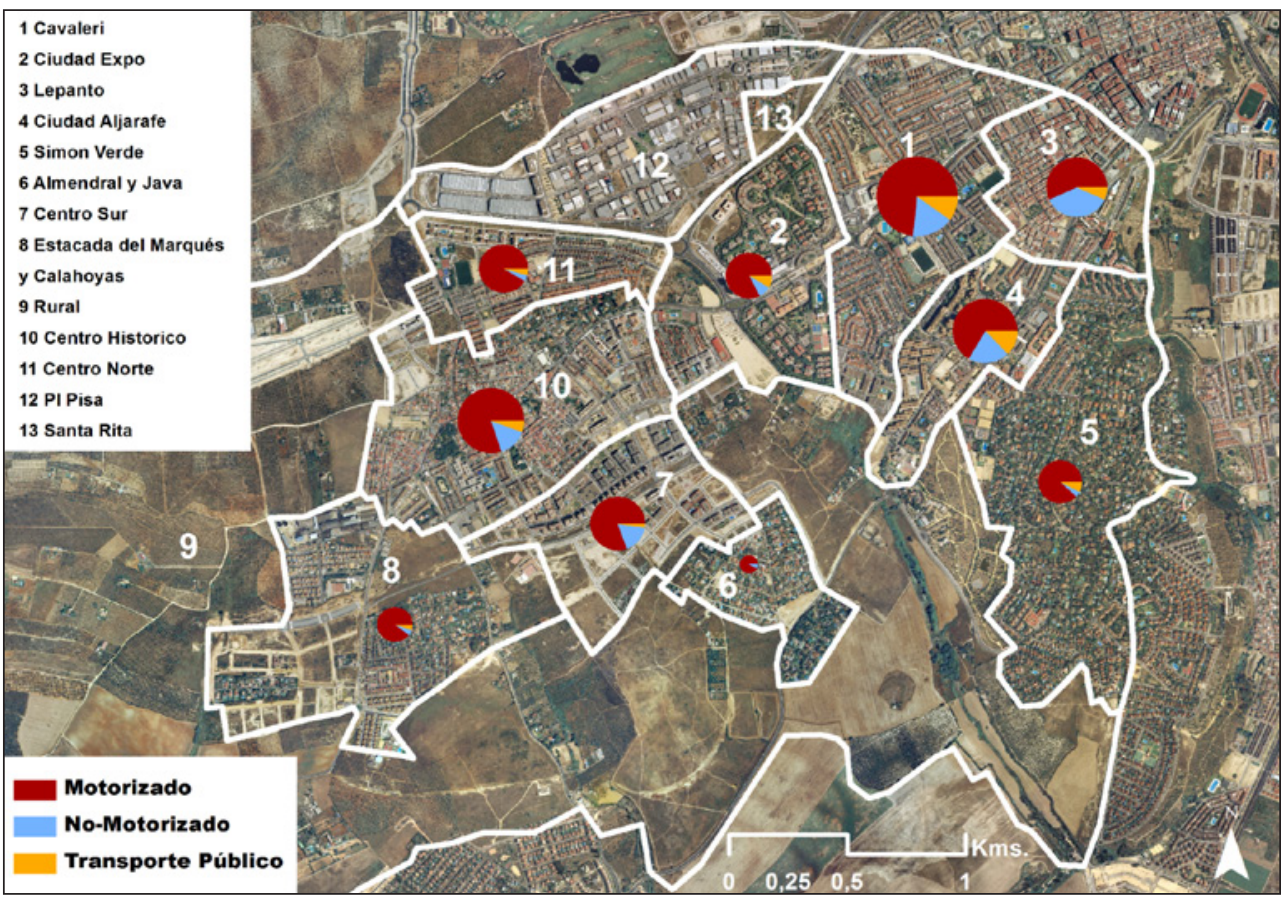

Fuente: elaboración propia. 
Igualmente, puede apreciarse cómo en las áreas con mayor dispersión el uso del transporte público es insignificante (Centro Sur, Estacada de Marqués y Calahoyas) o incluso inexistente (Almendral y Java). Cabe destacar cómo la población encuestada utiliza más el transporte no motorizado para sus desplazamientos al trabajo en las áreas con menor grado de dispersión urbana (como Lepanto, Ciudad Aljarafe y Cavaleri), respecto a las áreas más dispersas.

Para facilitar los análisis estadísticos se han agrupado las áreas según su nivel de dispersión (alto, medio y bajo). La tabla 3 presenta los valores $(\mathrm{N})$ y sus porcentajes (\%) para cada una de las categorías: modo de transporte y nivel de dispersión. Se observa cómo un $77,6 \%$ de los encuestados utiliza modos de transporte Motorizados para desplazarse hacia su trabajo, frente al 15,3\% que utiliza modos No-Motorizados y únicamente un 7,1\% Transporte Público. En dicha tabla se muestra igualmente la distribución de los encuestados según el grado de dispersión del área en la que residen. Se presentan 197 valores no contemplados debido a que se trata de población que no trabajaba, en el momento de realizar la encuesta, y que por tanto fue excluida de este análisis.

Tabla 3

RESUMEN DESCRIPTIVO DE LA DISTRIBUCIÓN DE LOS ENCUESTADOS SEGÚN EL MODO DE TRANSPORTE UTILIZADO PARA IR AL TRABAJO (MOTORIZADOS, NO - MOTORIZADOS Y TRANSPORTE PÚBLICO) YEL GRADO DE DISPERSIÓN URBANA

\begin{tabular}{|c|c|c|c|}
\hline & & $\mathbf{N}$ & Porcentaje \\
\hline \multirow{3}{*}{ Modo de Transporte al Trabajo } & Motorizados & 239 & $78 \%$ \\
\hline & No-Motorizados & 47 & $15 \%$ \\
\hline & Transporte Público & 22 & $7 \%$ \\
\hline \multirow{3}{*}{ Grado dispersión } & Bajo & 164 & $53 \%$ \\
\hline & Medio & 131 & $43 \%$ \\
\hline & Alto & 13 & $4 \%$ \\
\hline \multicolumn{2}{|l|}{ Válidos } & 308 & $100 \%$ \\
\hline \multicolumn{2}{|l|}{ Perdidos } & 197 & \\
\hline \multicolumn{2}{|l|}{ Total } & 505 & $100 \%$ \\
\hline
\end{tabular}

Fuente: elaboración propia.

En la Tabla 4 se puede apreciar como los valores observados son bastante similares (casi iguales) a los valores esperados obtenidos mediante la regresión ordinal, y que el modelo creado predice perfectamente el modo de transporte utilizado en función del grado de dispersión del área concreta en la que se reside. Tal y como se muestra en la tabla 5,34 encuestados que residían en áreas con bajo grado de dispersión utilizaban el trasporte no motorizado en sus desplazamientos diarios al trabajo (valor observado), siendo el valor esperado prácticamente el mismo $(34,008)$. De este modo, se verifica esta misma similitud en todos los casos, poniéndose de manifiesto cómo el modelo predice a la perfección la probabilidad de utilizar modos de transporte específicos en función del grado de dispersión en la que reside la población. 
Tabla 4

RELACIÓN ENTRE LOS VALORES OBSERVADOS Y LOS ESPERADOS

\begin{tabular}{|c|c|c|c|c|}
\hline \multirow{2}{*}{\multicolumn{2}{|c|}{ Grado dispersión urbana }} & \multicolumn{3}{|c|}{ Modos de Transporte } \\
\hline & & \multirow{2}{*}{$\begin{array}{c}\text { No-Motorizados } \\
1 \\
\end{array}$} & \multirow{2}{*}{$\begin{array}{c}\begin{array}{c}\text { Transporte } \\
\text { Público }\end{array} \\
0 \\
\end{array}$} & \multirow{2}{*}{$\begin{array}{c}\text { Vehículo Privado } \\
12 \\
\end{array}$} \\
\hline \multirow{3}{*}{ Alto } & Observada & & & \\
\hline & Esperada & 0,653 & 0,377 & 11,971 \\
\hline & Residuo de Pearson & 0,443 &,- 625 & ,001 \\
\hline \multirow{3}{*}{ Medio } & Observada & 12 & 7 & 112 \\
\hline & Esperada & 12,323 & 6,625 & 112,052 \\
\hline & Residuo de Pearson & $-0,097$ & ,149 & 0,013 \\
\hline \multirow{3}{*}{ Bajo } & Observada & 34 & 15 & 115 \\
\hline & Esperada & 34,008 & 14,989 & 115,003 \\
\hline & Residuo de Pearson & $-0,002$ & 0,003 & $-0,001$ \\
\hline
\end{tabular}

Fuente: elaboración propia.

Por último, es necesario destacar que la regresión logística con datos ordenados se basa en la hipótesis de que todas las categorías tienen odds proporcionales, es decir, que los efectos de las variables explicativas (en este caso, la dispersión urbana) se distribuyen de forma proporcional dentro de las diferentes categorías de la variable dependiente. Para corroborar el cumplimiento de esta hipótesis, se realizó el denominado test de líneas paralelas a través del estadístico Chi (ver tabla 5).

Tabla 5

PRUEBA DE LÍNEAS PARALELAS

\begin{tabular}{|l|c|c|c|c|}
\hline \multicolumn{1}{|c|}{ Modelo } & $\begin{array}{c}-\mathbf{2} \text { log de la } \\
\text { verosimilitud }\end{array}$ & Chi-cuadrado & gl & $\begin{array}{c}\text { Sig. } \\
\text { p valor }\end{array}$ \\
\hline Hipótesis nula & 20,424 & & & \\
\hline General & 14,5114 & 5,913 & 2 & 0,052 \\
\hline
\end{tabular}

Fuente: elaboración propia.

La hipótesis nula de este test es que los coeficientes son los mismos a lo largo de cada categoría. Como el modelo logit ordenado estima una sola ecuación sobre todos los niveles de las categorías, es necesario comprobar si esta asunción es correcta. Si se rechaza esta hipótesis nula, se debería concluir que este tipo de regresión no es adecuada bajo estas premisas. Sin embargo, el valor del p-valor (umbral de significación) es superior al 0,05, y no se debe contemplar la hipótesis de que fuera falsa esa premisa, por lo que este modelo es aplicable. El resultado de este test es el mismo que para el test de bondad de ajuste basado en el estadístico Chi-cuadrado, al haberse usado únicamente una variable explicativa. Dicho resultado diferiría si se le añadiesen más variables explicativas. La hipótesis nula establece que los parámetros de ubicación (los coeficientes para las pendientes) son los mismos para todas las categorías de respuesta. 


\section{DISCUSIÓN}

El uso de modos de transporte concretos depende de muchos factores, pero la probabilidad de usar un modo de transporte u otro, está asociada en parte con el grado de dispersión urbana. Otros estudios publicados, la mayoría en el ámbito anglosajón, han investigado la relación existente entre la dispersión urbana y el uso de los modos de transporte, aunque utilizando como ámbito de estudio la escala metropolitana. Resulta necesario extender la discusión sobre los efectos de la dispersión urbana también a otras escalas de mayor detalle, como la sub-municipal. Precisamente en esta investigación se ha mostrado de forma empírica la relación que existe entre el grado de dispersión urbana y el modo de transporte utilizado para los desplazamientos diarios a escala sub-municipal. Esta escala de detalle permite una mayor rigurosidad y precisión que los estudios realizados a escala metropolitana o incluso a escala municipal. Además, en este estudio se han utilizado un gran número de variables territoriales para establecer un índice compuesto que tiene en cuenta diversos factores territoriales.

El análisis estadístico realizado, utilizando, el modelo de regresión ordinal PLUM, ha demostrado que existe una relación entre la dispersión urbana y los modos de transporte utilizados. Los valores observados son bastante similares a los valores esperados, especialmente en niveles de dispersión bajo, lo que demuestra que el modelo predice con bastante certeza la probabilidad de utilizar modos de transporte específicos en función del grado de dispersión. Las personas que residen en áreas con alto grado de dispersión tienen mayor probabilidad de utilizar modos de transporte motorizados en sus desplazamientos diarios y son menos propensas a usar modos de transporte no-motorizados relacionados con la actividad física (bicicleta, a pie) que aquellos que residen especialmente en áreas con nivel de dispersión bajo y, en menor medida, medio.

El transporte relacionado con la actividad física, como caminar o montar en bicicleta, se considera actualmente como una solución sostenible, para resolver muchos de los problemas de salud pública y de transporte (Badland, Schofield, and Garrett 2008). Esta investigación corrobora que la dispersión urbana es un factor determinante para el uso del transporte relacionado con la actividad física, no sólo por parte de la población que reside en el ámbito anglosajón, sino que también condiciona la movilidad de la población que reside en ciudades mediterráneas.

\section{CONCLUSIONES}

Los resultados del presente estudio son consistentes con los resultados derivados de estudios previos descritos en la literatura científica. Esta investigación ha demostrado que la dispersión urbana condiciona la elección del modo de transporte a la hora de realizar los desplazamientos diarios, lo que repercute en diferentes facetas de la vida de la población y provoca un importante impacto sobre el medio ambiente. Las personas que residen en áreas urbanas con mayor grado de dispersión son más propensas a utilizar modos de transporte motorizados, que aquellas que residen en áreas de menor dispersión.

Las políticas urbanísticas deberían fomentar el desarrollo de áreas urbanas más compactas, favoreciendo la utilización de modos de transporte más sostenibles. Desde el punto de 
vista urbanístico, es necesario ordenar la expansión urbana, fomentando la mezcla de usos urbanos y la proximidad como base de accesibilidad. Desde la movilidad resulta necesario reducir la dependencia del automóvil, aumentar los desplazamientos no motorizados y recuperar el espacio público como lugar de encuentro y espacio de convivencia.

\section{AGRADECIMIENTOS}

Los resultados de este artículo forman parte del Proyecto de I+D "Evaluación del Impacto de la Dispersión Urbana en los Hábitos de Vida, la Movilidad Urbana y la Salud de la Población Adulta en Áreas Metropolitanas Españolas (URDIS)", financiado por el Ministerio de Economía y Competitividad de España (CSO2014-59524-P) y en el cual participan las Universidades de Sevilla y del País Vasco.

\section{BIBLIOGRAFÍA}

AKIN, O., MONTALVO, J. G., GARCÍA VIlLAR, J., PEYDRÓ, J. L. y RAYA, J. M. (2014): The real estate and credit bubble: evidence from Spain. SERIEs, 5(2-3), 223-243.

BAHRAINY, H. y KHOSRAVI, H. (2013): "The impact of urban design features and qualities on walkability and health in under-construction environments: The case of Hashtgerd New Town in Iran". Cities, 31, 17-28.

BRAÇE, O. (2016): Estudio de los efectos de la morfología urbana en la actividad física. Revista de Estudios Andaluces, 33(1), 24-39.

BROWN, B.B., SMITH, K.R., HANSON, H., FAN, J.X., KOWALESKI-JONES, L. y ZICK, C.D. (2013): "Neighborhood design for walking and biking: Physical activity and body mass index". American Journal of Preventive Medicine, 44(3), 231-238.

BRUEGMANN, R. (2005): Sprawl: A Compact History. Chicago: University of Chicago Press.

BURGESS, R. (2003): Ciudad y Sostenibilidad, Desarrollo urbano sostenible. Cuadernos de la CEPAL, 88, 193.

COMMISSION OF THE EUROPEAN COMMUNITIES (2004): Towards a Thematic Strategy on the Urban Environment. COM (2004) 60 Final, Bruxell.

DUANY, A., PLATER-ZYBERK, E. y SPECK, J. (2000): Suburban nation: the rise of sprawl and the decline of the American dream. New York: North Point Press.

EUROPEAN ENVIRONMENT AGENCY. (2006). Urban sprawl in Europe - The ignored challenge. (D. Ludlow, Ed.). Copenhagen: European Environment Agency.

EUROSTAT (2012): Europe in figures. Theme: General and regional statistics. Statistical books, Luxembourg: Publications Office of the European Union.

EWING, R. y CERVERO, R. (2001): “Travel and the Built Environmental: A Synthesis. Transportation Research Record". Journal of the Transportation Research Board, 1780(1), 87-114.

EWING, R., SCHMID, T., KILLINGSWORTH, R., ZLOT, A. y RAUDENBUSH, S. (2008): "Relationship between urban sprawl and physical activity, obesity, and morbidity". Urban Ecology: An International Perspective on the Interaction between Humans and Nature, 18(1), 567-582. 
EWING, R., MEAKINS, G., HAMIDI, S. y NELSON, A. C. (2014): Relationship between urban sprawl and physical activity, obesity, and morbidity - update and refinement. Health \& Place, 26, 118-26.

FRUMKIN, H., FRANK, L. y JACKSON, R. (2004): Urban sprawl and public health: Designing, planning, and building for healthy communities. Washington, DC: Island Press.

GÁLVEZ RUIZ, D., DIAZ CUEVAS, P., BRAÇE, O., GARRIDO-CUMBRERA, M. (2017): "Developing an index to measure sub-municipal level urban sprawl". Social Indicators Research, 135 (1). DOI: 10.1007/s11205-017-1801-3

GARCÍA PALOMARES, J.C. (2010): "Urban sprawl and travel to work: the case of the metropolitan area of Madrid”. Journal of Transport Geography, 18(2), 197-213.

GILES-CORTI, B., BULL, F., KNUIMAN, M., MCCORMACK, G., VAN NIEL, K., TIMPERIO, A. y BORUFF, B. (2013). The influence of urban design on neighbourhood walking following residential relocation: Longitudinal results from the RESIDE study. Social Science and Medicine, 77(1), 20-30.

JERRETT, M., ALMANZA, E., DAVIES, M., WOLCH, J., DUNTON, G., SPRUITJ-METZ, D. y PENTZ, M.A. (2013): "Smart growth community design and physical activity in children". American Journal of Preventive Medicine, 45(4), 386-392.

LÓPEZ LARA, E., MIRANDA BONILLA, J., POSADA SIMEÓN, J.C., NAVARRO LUNA, J., GARCÍA GÓMEZ, A. (2003): Servicios y transportes en el desarrollo territorial de España. Sevilla: Universidad de Sevilla.

MARMOLEJO DUARTE, C. R. y STALLBOHM, M. (2008): "En contra de la ciudad fragmentada ¿hacia un cambio de paradigma urbanístico en la Región Metropolitana de Barcelona?". Scripta Nova: Revista Electrónica de Geografía y Ciencias Sociales, 12(240), $1-16$.

NEWMAN, P. y KENWORTHY, J. (1999): Sustainability and Cities: Overcoming Automobile Dependence. Washington, DC: Island Press.

RODE, P., FLOATER, G., THOMOPOULOS, N., DOCHERTY, J., SCHWINGER, P., MAHENDRA, A., FANG, W. (2014): Accessibility in Cities: Transport and Urban Form. NCE Cities Paper 03. LSE Cities. London School of Economics and Political Science.

TRAVISI, C.M., CAMAGNI, R., y NIJKAMP, P. (2010): "Impacts of urban sprawl and commuting: a modelling study for Italy". Journal of Transport Geography, 18(3), 382-392.

UN-HABITAT. (2012): Prosperity of Cities: State of the World's Cities 2012/2013. State of the World's Cities. Routledge.

ZHAO, P., LÜ, B., y DE ROO, G. (2010): Urban expansion and transportation: the impact of urban form on commuting patterns on the city fringe of Beijing. Environment and Planning A, 42(10), 2467-2486. 
